\title{
Status of MEG experiment at PSI
}

\author{
Satoshi Mihara*† \\ High Energy Accelerator Research Organization (KEK), \\ Japan Proton Accelerator Research Complex (J-PARC), and \\ SOKENDAI \\ E-mail: satoshi.miharadkek.ip
}

The $\mu^{+} \rightarrow e^{+} \gamma$ decay is a key to understand physics beyond the standard model; if the decay is discovered, it is a clear evidence of new physics. If its branching ratio upper limit is improved by one order of magnitude, the result restricts the type of new physics in about twice larger energy scale. The MEG experiment, and its upgrade MEG II experiment, are experimental searches for the $\mu^{+} \rightarrow e^{+} \gamma$ decay by using intense DC muon beam provided at Paul Scherrer Institute in Switzerland. In this presentation we describe the result of MEG and report the preparation status of MEG II.

The 21st international workshop on neutrinos from accelerators (NuFact2019)

August 26 - August 31, 2019

Daegu, Korea

\footnotetext{
* Speaker.

${ }^{\dagger}$ on behalf of the MEG collaboration
} 


\section{Introduction}

The charged lepton-flavor violation (cLFV) is strictly forbidden in the standard model (SM). Even if we take the neutrino oscillation effect into account, the probability is extremely suppressed because of the tiny mass ratio of $m_{v}$ and $m_{W}$. Facts that we have not observed any muon reactions without emitting neutrinos in the final states and that the muon neutrino can produce only muon in its reaction led us to the idea of the flavor of elementary particles. On the other hand many of new physics models attempting to explain imperfection of the standard model by introducing new particles predict cLFV reactions, with rates just below current experimental bounds [W]. These reactions are $\mu^{+} \rightarrow e^{+} \gamma, \mu^{+} \rightarrow e^{+} e^{+} e^{-}$, and $\mu-e$ conversion in muonic atoms for muons, and $\tau^{ \pm} \rightarrow \mu^{ \pm} \gamma$, $\tau^{ \pm} \rightarrow \mu^{ \pm} \mu^{ \pm} \mu^{\mp}, \tau^{ \pm} \rightarrow e^{ \pm} \gamma$, and $\tau^{ \pm} \rightarrow e^{ \pm} e^{ \pm} e^{\mp}$ and others including hadrons in the final states for tau leptons. Among experimental attempts to find any of these, the $\mu^{+} \rightarrow e^{+} \gamma$ search is thought to have high sensitivity because highly intense muon beam is available at high-power proton accelerator facilities, and muons have sufficiently long life time to carry out an experiment in a laboratory. It is also good that there are two particles in the final state, allowing us to develop multi-parameter analysis to distinguish the signal from the background although the experiment sensitivity could be limited by accidental overlaps in the final state from different sources. At the moment there are intensive activities in the world to search for muon cLFV reactions; $\mu^{+} \rightarrow e^{+} \gamma$ decay search at PSI (MEG II) [व], $\mu^{+} \rightarrow e^{+} e^{+} e^{-}$decay search at PSI (Mu3e) [3] , and $\mu-e$ conversion searches at J-PARC (COMET [四] and DeeMe [回]) and at FNAL (Mu2e) [四]. Comparison of their results provides us precious information to understand the physics behind the phenomena once they are observed experimentally.

\section{MEG}

The MEG experiment at PSI has been a leading experiment after previous experimental attempts to search for $\mu^{+} \rightarrow e^{+} \gamma$ (the MEGA experiment at LANL) [四] and $\mu-e$ conversion (the SINDRUM II experiment at PSI) [U] completed. The MEG experiment employed novel techniques to identify gammas and positrons from $\mu^{+} \rightarrow e^{+} \gamma$ decays [8]; the energy, position, and arrival timing of gammas was measured by a liquid-xenon photon detector with scintillation light readout using photomultipliers (PMTs) submersed in the liquid, and the positron momentum along with the emission direction from the muon stopping target was measured by low-mass segmented drift chambers arranged in a solenoidal magnetic field. The positron timing was measured by bar scintillation counters with fine-mesh PMTs operational in magnetic field. The data acquisition of MEG was completed in 2013. The final result was released to report the $90 \%$ C.L. upper limit of $4.2 \times 10^{-13}$ [Q] .

\section{MEG II}

Immediately after the MEG experiment completed its data acquisition, the collaboration started to work on improving the physics sensitivity by one order of magnitude. This will be achieved by realizing better detector resolutions by about a factor of two in all detector components and by increasing their detection efficiencies and muon beam intensity used for the experiment. 


\subsection{Photon Detector}

The liquid-xenon photon detector used in MEG has been upgraded by replacing the photosensors used on the front face of the detector where gamma ray traverses before entering the active volume. A specially developed silicon photomultipliers (SiPMs) for the use to detect xenon scintillation light replaced 2-inch PMTs on the front face. Total of $4092 \mathrm{SiPMs}$ covers the front face while 216 photomultipliers were used in MEG. The size of the SiPM is $12 \times 12 \mathrm{~mm}^{2}$, allowing us to achieve better background rejection thanks to better position resolution; accidental overlaps of two photon incident to the detector will be more clearly identified in this new detector configuration. A new Gilfford-McMahon (GM) refrigerator system has also been introduced to accommodate higher heat load through more signal readout cables newly installed for SiPMs.

\subsection{Positron Tracker}

A new cylindrical drift chamber (CDC) is installed in MEG II to reconstruct the positron track in the spectrometer magnet. The $\mathrm{CDC}$ has a single volume with higher granularity of sense wires to achieve better angular and momentum resolutions compared to the segmented drift chambers employed in MEG. Higher transmission of positrons to the timing counters is also achieved thanks to the single active volume with a thin outer wall. Stereo wires are used to reconstruct the hit position along the axial direction of the CDC. A new gas mixture of $\mathrm{He}$ and isobutane $\left(\mathrm{C}_{4} \mathrm{H}_{10}\right)$ in the ratio of 90:10 is used to realize an equivalent radiation length along a positron track of about $1.58 \times 10^{-3} \mathrm{X}_{0}$ (compared with $2.0 \times 10^{-3}$ in the MEG drift chamber using a gas mixture of $\mathrm{He}$ and ethene $\left(\mathrm{C}_{2} \mathrm{H}_{4}\right)$ in the ratio of 50:50).

\subsection{Positron Timing Counter}

The positron timing counter is completely modified from that used in MEG. In total 256 pixelated plastic scintillator with SiPM readout replaces 15 scintillator bar counters on one end of the spectrometer. This configuration provides us multi-counter hits in one single positron track, allowing us to achieve better timing resolution as good as $30 \mathrm{psec}$. A cooling system is adopted to stabilize the SiPM temperature, which is indispensable to operate SiPMs stably.

\subsection{Radiative Decay Counter}

A new detector system is introduced in MEG II to suppress the number of radiative muon decay events with a high-energy gamma entering the photon detector in the final data sample. Such event possibly overlaps with a Michel decay event with a high-energy positron emission to the positron spectrometer, resulting in mimicking the signal event. The high-energy gamma is usually generated in a radiative muon decay including a low-energy positron, which does not leave any hit in the positron tracker, and thus fails to be identified in the analysis algorithm as a background. Two different type detectors are designed to identify such low-energy positrons at both ends of the spectrometer magnet. At the downstream side of the muon beam, a hybrid detector composed of an array of LYSO crystals with SiPM readout to measure the positron energy and plastic scintillation detector with SiPM readout to measure its arrival timing is installed. At the upstream side of the muon beam, an R\&D program is in progress to realize the similar detection of the positron but with less amount of material without losing the detection efficiency.

A schematic view of the MEG II setup is shown in Figure $\mathbb{W}$. 


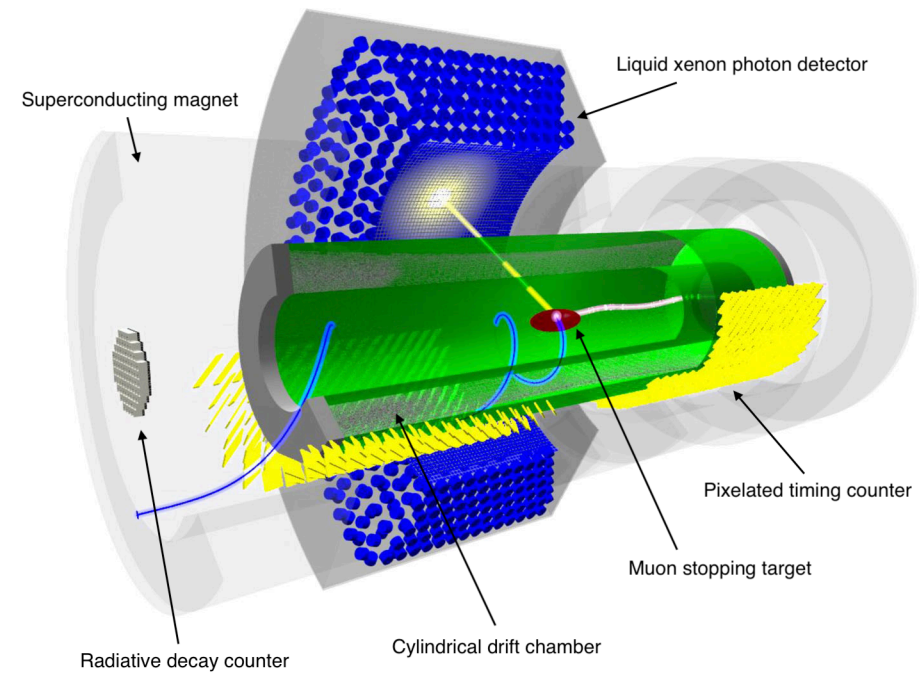

Figure 1: Schematic view of the MEG II experiment at PSI.

\subsection{Expected Sensitivity}

Based on prototype tests and detailed detector simulations, expected resolutions and detection efficiencies of each detector components are estimated as summarized in Tablell. Significant improvements in detection efficiencies are expected thanks to replacing photosensors on the photon detector front face and modifying the positron spectrometer geometry. Improvement of the timing resolution can be achieved owing to excellent performance of SiPMs and better segmentation both in the photon detector and positron timing counter.

The sensitivity of the MEG II experiment is $6 \times 10^{-14}$ for $90 \%$ C.L. exclusion with 3 years data acquisition. Discovery potential at $5 \sigma$ level is $2 \times 10^{-13}$ with the same amount of data statistics.

\subsection{Status of the Experiment}

The MEG II is in the phase of detector preparation and conditioning as of summer 2019. Construction of the CDC completed in 2018, followed by conditioning on site in 2019 fall. The positron timing counter was fabricated in 2017 and its basic performance test with muon beam has already been completed. The liquid-xenon photon detector is in position beside the spectrometer magnet with the cryogenic facility connected. Conditioning with liquid xenon has already been completed and performance evaluation with new SiPMs is in progress using gamma rays produced with muon beams. The newly fabricated RDC was installed in 2017 at the downstream side only and initial test of its performance has already been completed. New DAQ system integrating waveform digitizers and trigger electronics are being installed in early 2020.

\section{Summary}

The MEG experiment at PSI has established the most stringent limit on the $\mu^{+} \rightarrow e^{+} \gamma$ decay at 
Table 1: Comparison of measurement resolutions and efficiencies between MEG and MEG II. $u_{\gamma}, v_{\gamma}$, and $w_{\gamma}$ are coordinate variables along the inner surface of the photon detector; $u_{\gamma}$ parallel to the beam, $v_{\gamma}$ perpendicular to $u_{\gamma}$ on the curved inner surface, and $w_{\gamma}$ along the depth from the surface.

\begin{tabular}{lccc}
\hline Resolution & & MEG & MEG II \\
\hline Positron Energy & $E_{e^{+}}(\mathrm{keV})$ & 380 & 130 \\
Positron Polar Angle & $\theta_{e^{+}}(\mathrm{mrad})$ & 9.4 & 5.3 \\
Positron Azimuthal Angle & $\phi_{e^{+}}(\mathrm{mrad})$ & 8.7 & 3.7 \\
Positron Decay Vertex & $z_{e^{+}} / y_{e^{+}}(\mathrm{mm}) \mathrm{core}$ & $2.4 / 1.2$ & $1.6 / 0.7$ \\
Photon Energy & $E_{\gamma}(\%)(\mathrm{w}>2 \mathrm{~cm} /<2 \mathrm{~cm})$ & $1.7 / 2.4$ & $1.0 / 1.1$ \\
Photon Interaction Position & $u_{\gamma}, v_{\gamma}, w_{\gamma}(\mathrm{mm})$ & $5 / 5 / 6$ & $2.6 / 2.2 / 5$ \\
Relative timing between positron and gamma & $t_{e \gamma}(\mathrm{psec})$ & 122 & 84 \\
\hline Efficiency $(\%)$ & & & \\
\hline Trigger & 99 & 99 & \\
$\gamma$ & 63 & 69 & \\
$e^{+}$(tracking $\times$matching) & 30 & 70 & \\
\hline
\end{tabular}

$4.2 \times 10^{-13}$ at $90 \%$ C.L. This is an extreme constraint on the possibility of new physics beyond the standard model. Upgrade of the experiment, MEG II, is in preparation to achieve almost 10 times better sensitivity $\left(6 \times 10^{-14}\right.$ at $90 \%$ C.L. $)$ by improving detector resolutions and efficiencies. The MEG II will be conducted at the PiE5 beam line at PSI, where the MEG experiment was carried out, with 2 times larger muon intensity. Engineering run of the MEG II experiment continues in 2019 toward the start of physics data acquisition in early 2020.

\section{References}

[1] S. Mihara, J. Miller, P. Paradisi and G. Piredda, Ann. Rev. of Nucl. and Part. Sci. 63 (2013) 531, doi:10.1146/annurev-nucl-102912-144530.

[2] A. M. Baldini et al., Euro. Phys. J. C 78:380, doi:10.1140/epjc/s10052-018-5845-6.

[3] A. Blondel et al., arXiv:1301.6113 (2013).

[4] R. Abramishvili et al. (COMET Collaboration), http://comet.kek.jp/Documents files/PAC-TDR-2016/COMET-TDR-2016 v2.pdf.

[5] L. Bartoszek et al (Mu2e Collaboration), arXiv:1501.05241 (2015).

[6] M. Aoki, 19th Particle and Nuclei International Conference, AIP Conference Proceedings 1441, 599 (2012), doi:10.1063/1.3700628.

[7] M. Ahmed et al., Phys. Rev. D 65 112002, doi:10.1103/PhysRevD.65.112002.

[8] J. Adam et al., Euro. Phys. J. C 73:2365, doi:10.1140/epjc/s10052-013-2365-2.

[9] A. M. Baldini et al., Euro. Phys. J. C 76:434, doi:10.1140/epjc/s10052-016-4271-x.

[10] W. Bertl et al., Euro. Phys. J. C 47:337, doi:10.1140/epjc/s2006-02582-x. 